\title{
Changes of Serum Alkaline Phosphatase Isoenzymes in Fasted Rats
}

\author{
Hiroshi WADA, ${ }^{1,3, *}$ Noboru Niwa, ${ }^{2}$ Takashi HAYAKAwA, ${ }^{2}$ \\ and Haruhito TSUGE ${ }^{2}$ \\ ${ }^{1}$ The United Graduate School of Agricultural Science, and \\ ${ }^{2}$ Department of Food Science, Faculty of Agriculture, \\ Gifu University, Gifu 501-11, Japan \\ ${ }^{3}$ Pharmaceutical Division, Ciba Geigy Japan, \\ Takarazuka 665, Japan
}

(Received April 23, 1996)

\begin{abstract}
Summary Changes of serum alkaline phosphatase (sALP) isoenzymes under fasting conditions were examined using polyacrylamide gel electrophoresis (PAGE), amino-acids (L-phenylalanine (L-Phe), L-homoarginine (L-HArg) ) inhibition and wheat germ agglutinin (WGA) treatment. The SALP of non-fasted rats was separated into three bands (S1, S2, S3) by PAGE. The molecular weight (M.W.) of S1 corresponded to that of an isoenzyme found in the ileum. By the addition of L-Phe, the staining intensity of S1 was weakened, S2 and S3 remained unchanged and the total activity of the isoenzymes extracted from intestine decreased. On the other hand, the activity of isoenzymes extracted from kidney and bone decreased by the addition of L-HArg. Therefore, S1 was judged to be derived from intestine. The activities of total SALP and S1 decreased from $16 \mathrm{~h}$ of fasting. Total sALP activity and SALP activity of the supernatant prepared by WGA treatment decreased, whereas the ALP activity of the precipitate (difference between total SALP activity and supernatant SALP activity) did not change. The activity band of the precipitate corresponded to that of S3 by PAGE. Therefore, S3 was judged to be derived from bone. In conclusion, under fasting conditions, the activity of S1 decreased while the activities of S2 and S3 remained unchanged.
\end{abstract}

Key Words serum alkaline phosphatase, fasting, rat, polyacrylamide gel electrophoresis

* To whom correspondence should be addressed.

Abbreviations: ALP, alkaline phosphatase; sALP, serum alkaline phosphatase; WGA, wheat germ agglutinin; PAGE, polyacrylamide gel electrophoresis; PNPP, disodium $p$ nitrophenyl phosphate; L-Phe, L-phenylalanine; L-HArg, L-homoarginine; GlcNAc, Nacetyl-D-glucosamine; M.W., molecular weight; tALP, tissue alkaline phosphatase; SDS, sodium dodecyl sulfate. 
The ALP [orthophosphoric monoester phosphohydrolase (alkaline optimum) EC 3.1.3.1] is a group of enzymes which catalyze the hydrolysis of phosphate esters at an alkaline pH. It is well known that isoenzymes of ALP are distributed in liver, bone, kidney, placenta, small intestine, etc. in humans (1), and they are considered to be related to the fundamental functions of living bodies. ALP activity is also found in serum (2).

The activity of sALP has been determined as the total sum of activities of these types of ALP, however, the analysis of ALP isoenzymes originating from each organ is very important from the diagnostic viewpoint. Electrophoresis (3), thermostability (4), amino-acid inhibition $(5,6)$, neuraminidase digestion (7), WGA treatment (8), immunological technique (9) and gel filtration (10) have been used for the separation of SALP isoenzymes in humans.

Concerning the separation of rat sALP isoenzymes, many efforts have been made (11-13). Electrophoretic analysis is a useful method and was therefore chosen from among the above-mentioned methods.

The number of bands of ALP activity demonstrated in rat serum would appear, from literature, to depend on the techniques used in the separation. Kaschnitz et al. reported that PAGE revealed two bands in normal rats (14), whereas there is another report contradicting this finding (15). Additionally, since rat sALP changes according to nutritional conditions $(16,17)$, to clarify the change in SALP under fasting conditions seems to be very important. Therefore, the purpose of this study is to inquire into the processes of separating rat sALP isoenzymes by PAGE and to examine their changes caused by fasting.

\section{MATERIALS AND METHODS}

Materials. PNPP, L-Phe and L-HArg were purchased from NACALAI TESQUE, INC. (Kyoto, Japan), 5-bromo-4-chloro-3-indolyl "phosphate, ptoluidine salt, GlcNAc and WGA were from Sigma Chemical Co., Ltd. (St. Louis, MO. USA), the M.W. estimation standard kit was from Bio-Rad Laboratories (SDS-PAGE Protein Standards, Hercules, CA, USA), and Epoxy-activated Sepharose $6 \mathrm{~B}$ was from Pharmacia Biotech AB (Uppsala, Sweden). All other chemicals were of analytical grade from NACALAI TESQUE, INC.

Animals. Male Sprague-Dawley rats weighing $160-180 \mathrm{~g}$ at the age of six weeks (Charles River Japan, Yokohama, Japan) were used after one week acclimation to the breeding environment. They had free access to a chow diet (CE-2, Clea Inc., Tokyo) except for the period of fasting, and they had free access to water throughout the experiment. The temperature and humidity of the animal room were controlled at $22 \pm 1^{\circ} \mathrm{C}$ and $55 \pm 5 \%$, respectively. A $12 \mathrm{~h}$ light/dark cycle was used throughout the experiment (light period, 0700-1900). Twelve rats were allocated to the "fasted group."

Preparation of serum and tissue marker. After acclimation, the fasted group was fasted during the experiment. Blood was taken before fasting, and 16, 24 and 
$40 \mathrm{~h}$ after fasting. Food was removed at 7:00 a.m. Blood samples were taken from the orbital venous plexus under light anesthesia with ether. Serum was separated by centrifugation at $1,500 \times g$, for $15 \mathrm{~min}$ and stored at $-20^{\circ}$ until used. In order to prepare the ALP enzymes extracted from each tissue, the fractions of ALP from rat liver, kidney, bone (femur) and intestine (duodenum, jejunum, ileum) were used. tALP was extracted according to the method of Saini and Done (18). Each tissue was immediately homogenized in five volumes of $10 \mathrm{~mm}$ Tris- $\mathrm{HCl}$ buffer ( $\mathrm{pH} \mathrm{8.0)}$, containing $1 \mathrm{mM}$ phenylmethyl sulphonyl fluoride, $1 \mathrm{~mm}$ benzamidine hydrochloride and $0.5 \%$ Triton $\mathrm{X}-100$, at $4{ }^{\circ} \mathrm{C}$ for $1 \mathrm{~min}$ in a Waring Blender. The homogenates were treated with $30 \%(\mathrm{v} / \mathrm{v}) n$-butanol, and the resulting suspension was centrifuged at $15,000 \times g$ for $30 \mathrm{~min}$ at $4{ }^{\circ} \mathrm{C}(1,8)$. The aqueous layer was stored at $-20^{\circ} \mathrm{C}$ until used. The ALP activity and protein concentration of the tissues were assayed as described below. The specimens were defrosted on the day of use and tested immediately. The specific activities of ALP extracted from each tissue were $0.002 \mathrm{U} / \mathrm{mg}$ for liver, $1.165 \mathrm{U} / \mathrm{mg}$ for kidney, $1.826 \mathrm{U} / \mathrm{mg}$ for bone, $3.152 \mathrm{U} / \mathrm{mg}$ for duodenum, $0.648 \mathrm{U} / \mathrm{mg}$ for jejunum and $0.229 \mathrm{U} / \mathrm{mg}$ for ileum.

Enzyme assay and protein content

Assay of ALP activity: \{tALP, sALP, L-Phe (or L-HArg) insensitive tALP, L-Phe (or L-HArg) insensitive sALP, bone type SALP $\}$ : ALP activity was assayed at $37^{\circ} \mathrm{C}$ with PNPP as the substrate by the method of Bessey-Lowry (19). Enzyme activity was expressed in terms of international units (U). One unit (U) is defined as the enzyme activity that catalyzes the hydrolysis of one $\mu \mathrm{mol}$ of PNPP per min under standard conditions. Concerning L-Phe or L-HArg insensitive tALP activity, L-Phe $(20 \mathrm{mM})$ or L-HArg $(10 \mathrm{mM})$ was added to the reaction mixture of the tALP activity assay (20). The residual ALP activity was assayed. L-Phe or L-HArg insensitive SALP activity was assayed in a manner similar to L-Phe or L-HArg insensitive tALP activity. For the assay of bone-type sALP activity, WGA was purified in our laboratory according to the method of Vretblad (21) with crude WGA purchased from Sigma. WGA $(150 \mu \mathrm{l})$ in a concentration of $5 \mathrm{mg} / \mathrm{ml}$ in 150 $\mu \mathrm{l}$ of bovine serum albumin solution $(50 \mathrm{mg} / \mathrm{ml})$ was added to rat serum $(150 \mu \mathrm{l})$. After incubation at $37^{\circ} \mathrm{C}$ for $30 \mathrm{~min}$ and centrifugation at $37^{\circ} \mathrm{C}$ for $10 \mathrm{~min}$ at $15,000 \times g$, the ALP activity in the supernatant fluid was determined. The total SALP activity, which was obtained without the addition of WGA, was also determined. The bone-type SALP activity was calculated by subtracting the supernatant ALP activity from the total SALP activity.

Protein content: Tissue protein concentration was determined by the method of Lowry et al. (22) using bovine serum albumin as the standard.

$P A G E$. PAGE was conducted with sera and the second peak of protein eluate of GlcNAc epoxy-activated Sepharose 6B column chromatography. Samples of sera before fasting, and 16, 24 and $40 \mathrm{~h}$ after fasting were electrophoresed. PAGE with Ready Gels J (5-10\%, Bio-Rad. Lab.) was performed under nonreducing conditions (23) using a BIO-RAD Mini-Protean II Electrophoresis Cell Apparatus (Bio-Rad Lab.) with $90 \mathrm{~mm}$ Tris/borate-SDS (0.1\%) buffer ( $\mathrm{pH} 9.0)$ in the buffer 
compartments. ALP activity was not inhibited by SDS at the final concentration up to at least 3\% (data not shown). Fixed amount of samples $(0.5-20 \mu \mathrm{l})$ in each experiment were electrophoresed at $10 \mathrm{~mA} / \mathrm{gel}$ for $180 \mathrm{~min}$ at $4^{\circ} \mathrm{C}$, and the staining for ALP activity was applied.

ALP activity staining. ALP activity was demonstrated after electrophoresis by incubating the gel for $60 \mathrm{~min}$ at $37^{\circ} \mathrm{C}$ with a chromogenic substrate, 5-bromo-4chloro-3-indolyl phosphate, $p$-toluidine salt at a final concentration of $1.25 \mathrm{~mm}$ in a 2-amino-2-methyl-1,3-propanediol buffer $(0.1 \mathrm{M}, \mathrm{pH} 10.2)$ and magnesium sulfate (1 mM) (24). The stained gel was then soaked for $5 \mathrm{~min}$ in aqueous acetic acid (50 $\mathrm{ml} /$ liter), washed with distilled water and dried.

Protein staining. Gels, on which the M.W. standards were electrophoresed, were fixed for $60 \mathrm{~min}$ with $12.5 \%$ trichloroacetic acid. The fixed gels were then stained for $60 \mathrm{~min}$ in aqueous CBB R-250 methanol/acetic acid and decolorized in $7.5 \%$ acetic acid for $24 \mathrm{~h}(9)$.

Amino acid inhibition. To assay the activity of L-Phe insensitive SALP isoenzyme bands on polyacrylamide gel, L-Phe $(20 \mathrm{~mm})$ was added to the activity staining solution as mentioned above. Other methods were the same as described for ALP activity staining.

Determination of M.W. The M.W. of serum and tissue isoenzymes were calculated from the obtained M.W. standards curve of the SDS-PAGE High-Range Protein Standards (Bio-Rad. Lab.).

Densitometric analysis of electrophoretograms. The ALP isoenzymes on polyacrylamide gel were analyzed using an Image Analyzer V1 (Toyobo, Osaka, Japan).

Separation of $A L P$ protein from the $W G A-A L P$ complex. A GlcNAc epoxyactivated Sepharose $6 \mathrm{~B}$ column $(2.0 \times 6.5 \mathrm{~cm})$ was prepared according to the method of Vretblad (21) by using epoxy-activated Sepharose $6 \mathrm{~B}$ to separate ALP protein from the precipitate (WGA-ALP complex) prepared by WGA. The column was equilibrated with $0.05 \mathrm{M}$ sodium acetate buffer $(\mathrm{pH} 4.5)$. The precipitate was dissolved with $400 \mu 1$ of $0.05 \mathrm{M}$ sodium acetate buffer ( $\mathrm{pH} 4.5$ ) and applied to the column. The column was then washed with the same buffer solution. The protein having ALP activity (ALP protein) was released with a linear gradient of $0-0.1 \mathrm{M}$ GlcNAc solution.

Statistical analysis. For statistical analysis of differences in the changes in rat sALP activities with or without amino acids inhibition, one-way repeated measurement analysis of variance (ANOVA) was performed, followed by Scheffe's test for multiple comparisons where appropriate. Concerning L-Phe or L-HArg insensitive tALP activities, densitometric analysis values of SALP isoenzyme bands and rat sALP activities by WGA treatment, Duncan's multiple-range test was performed among means. Data are expressed as $\mathbf{M} \pm$ SEM. A probability within 0.05 was considered to indicate significant difference. 


\section{RESULTS}

\section{SALP and $t A L P$}

Changes in rat SALP activities with or without amino-acid inhibition. The time courses of SALP activity, SALP activity with L-Phe (L-Phe insensitive sALP activity) and SALP activity with L-HArg (L-HArg insensitive SALP activity) are shown in Fig. 1. sALP activity decreased significantly at $16 \mathrm{~h}$ of fasting and remained at a constant level thereafter. L-Phe insensitive SALP activity remained at a constant level and was not influenced. However, L-HArg insensitive sALP activity decreased at $16 \mathrm{~h}$ of fasting and remained at a constant level thereafter.

Various tALP activities insensitive to L-Phe or L-HArg. The results are shown in Fig.2. With the addition of L-Phe $(20 \mathrm{mM})$, the tALP activities of the duodenum, jejunum and ileum were significantly inhibited. On the other hand, with the addition of L-HArg (10 $\mathrm{mM})$, the tALP activities of the duodenum, jejunum and ileum were not inhibited, while the tALP activities of the kidney and bone were inhibited more than $60 \%$ in the control group.

\section{$P A G E$}

Electrophoretograms of rat SALP by fasting. The gel stained for ALP activity is shown in Fig. 3, and the results of the densitometric analysis of Fig. 3 are shown in Fig. 4. The serum before fasting was separated into three bands (S1, S2, S3) by PAGE. Concerning the time course of electrophoretograms after fasting, the activity of $\mathrm{S} 1$ decreased after $16 \mathrm{~h}$ of fasting. As it has been reported that fasting decreases intestinal-type sALP activity and feeding increases intestinal-type sALP activity (16), the origin of S1 was thought to be in the intestine. The activities of

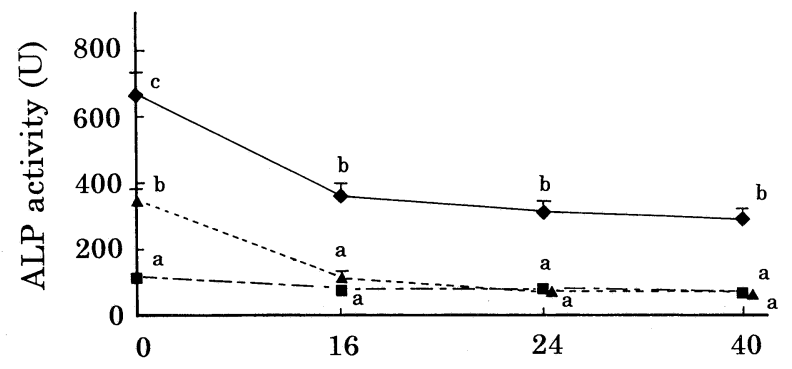

Fasting period (hr)

Fig. 1. Changes in rat SALP activities with or without amino-acid inhibition.

control, 1 L-Phe, $\triangle$ L-HArg. Control: tota SALP activity without amino-acid inhibition, L-Phe: SALP activity with L-Phe $(20 \mathrm{mM})$ (L-Phe insensitive sALP activity), L-HArg: sALP activity with L-HArg (10 mM) (L-HArg insensitive sALP activity). Each point indicates the $M \pm S E M$ of 5-6 rats. Points without a common superscript letter in the same line are significantly different $(p<0.05)$. 


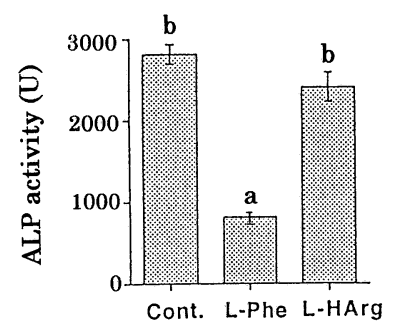

(Duodenum)

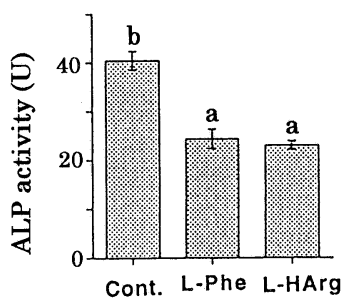

(Liver)

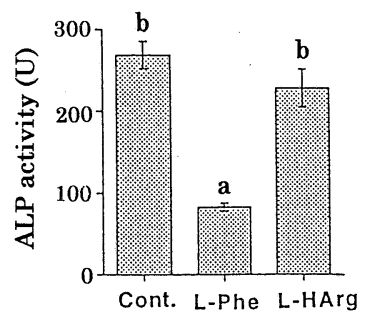

(Jejunum)

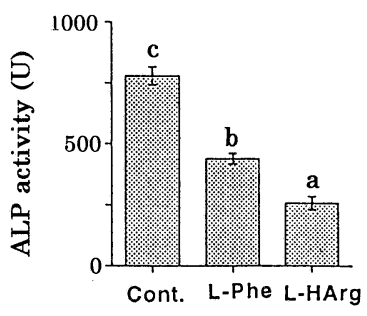

(Kidney)

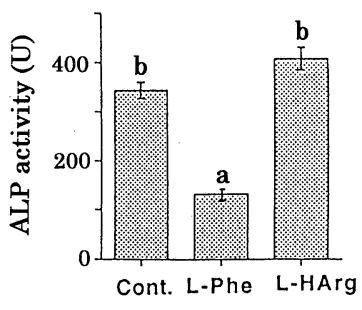

(Ileum)

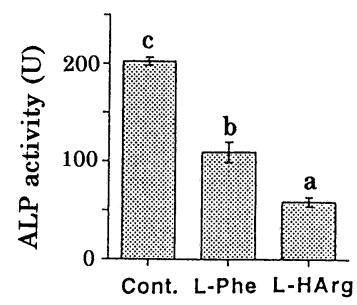

(Bone)

Fig. 2. Various tALP activities insensitive to L-Phe or L-HArg. Cont.: tALP activity without the addition of L-Phe or L-HArg, L-Phe: L-Phe insensitive tALP activity, L-HArg: L-HArg insensitive tALP activity. Each value indicates the $\mathrm{M} \pm \mathrm{SEM}$ of five determinations. Bars without a common superscript letter in the same graph are significantly different $(p<0.05)$.

S2 and S3 were not changed under fasting conditions.

M.W. of ALP isoenzymes and activity ratios of isoenzymes in rat serum and tissues. The activities of the bands were determined using an Image Analyzer V1, and their activity rates were calculated. The M.W. and activity ratios of the isoenzymes are shown in Table 1. The M.W. of S1 corresponded to that of I1. Neither the M.W. of S2 nor S3 corresponded to any isoenzymes derived from the tissues.

Electrophoretograms of rat $S A L P$ by fasting with or without L-Phe. The gel stained for ALP activity is shown in Fig. 5(A), and the densitometric analysis of serum before fasting with L-Phe is shown in Fig. 5(B). The staining intensity of S1 was eliminated by the addition of L-Phe. The staining intensities of S2 and S3 were not changed. As the M.W. of S1 corresponded to that of I1 and it is reported that L-Phe inhibits the isoenzymes of intestinal-type ALP (25), the component (S1) eliminated first, is considered to be an isoenzyme of the intestine.

Changes in total SALP and SALP activities precipitated by WGA treatment after 


\section{Fasting period (hr)}
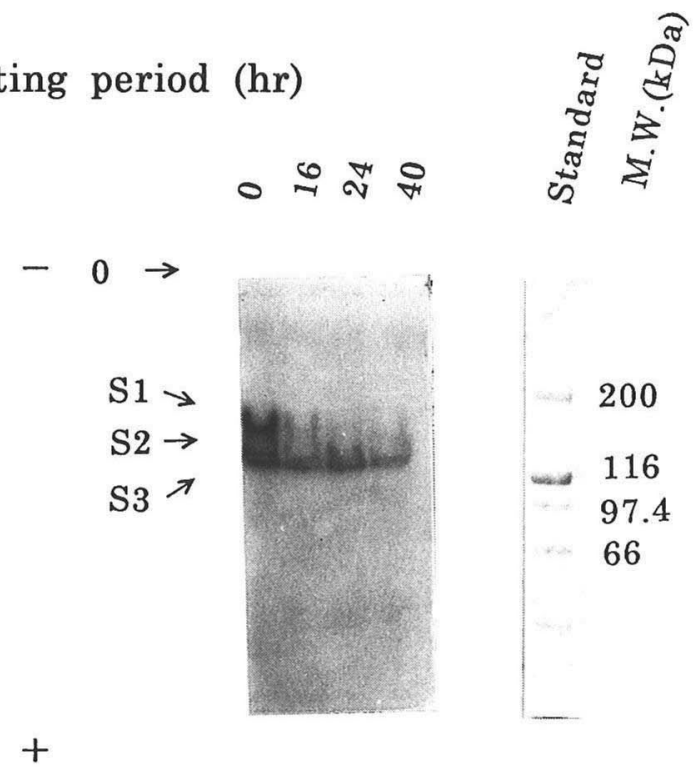

Fig. 3. Electrophoretograms of rat sALP by fasting. The left gel was stained for ALP activity and the right gel for standard M.W. was stained for protein.

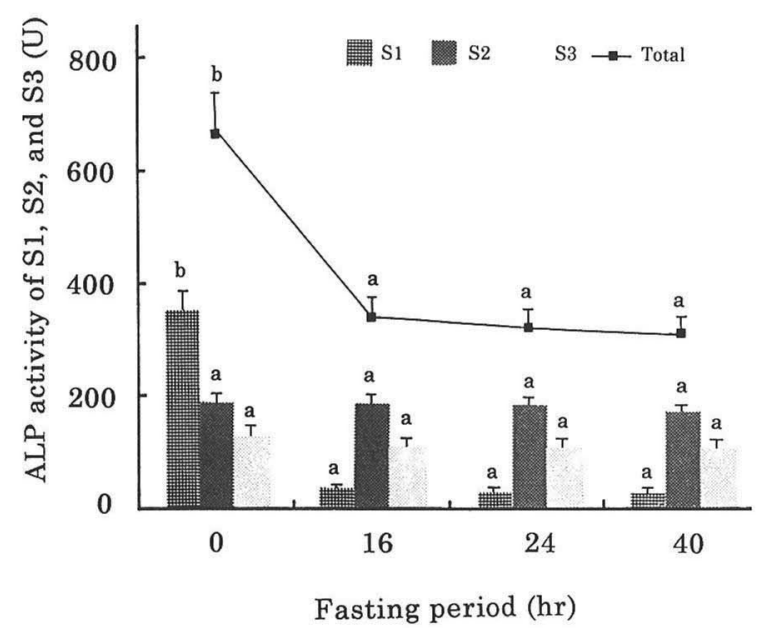

Fig. 4. Changes in SALP isoenzyme activities in rat by densitometric analysis reported in Fig. 3. S1: ALP activity of the S1 band, S2: ALP activity of the S2 band, S3: ALP activity of the S3 band, Total: total SALP activity. The isoenzyme activities were calculated using the activity ratios of isoenzymes, which were determined using an Image Analyzer V1, and sALP activities. Each value indicates the $\mathrm{M} \pm \mathrm{SEM}$ of $3-5$ determinations. Bars without a common superscript letter in the same type of graph are significantly different $(p<0.05)$. 
Table 1. Molecular weights of ALP isoenzymes and activity ratios of isoenzymes in rat serum and tissues.

\begin{tabular}{lccc}
\hline Tissue & $\begin{array}{c}\text { Isoenzyme } \\
\text { number }\end{array}$ & $\begin{array}{c}\text { Molecular weight } \\
(\mathrm{kDa})\end{array}$ & $\begin{array}{c}\text { Activity ratio } \\
(\%)\end{array}$ \\
\hline Liver & $\mathrm{L}_{1}$ & $166 \pm 2$ & 47 \\
& $\mathrm{~L}_{2}$ & $110 \pm 1$ & 53 \\
Kidney & $\mathrm{K}_{1}$ & $163 \pm 1$ & 52 \\
& $\mathrm{~K}_{2}$ & $119 \pm 1$ & 48 \\
Bone & $\mathrm{B}_{1}$ & $110 \pm 2$ & 100 \\
Duodenum & $\mathrm{D}_{1}$ & $160 \pm 4$ & 16 \\
& $\mathrm{D}_{2}$ & $135 \pm 2$ & 84 \\
Jejunum & $\mathrm{J}_{1}$ & $131 \pm 2$ & 54 \\
& $\mathrm{~J}_{2}$ & $114 \pm 2$ & 46 \\
Ileum & $\mathrm{I}_{1}$ & $188 \pm 2$ & 48 \\
& $\mathrm{I}_{2}$ & $114 \pm 1$ & 52 \\
Serum & $\mathrm{S}_{1}$ & $188 \pm 1$ & 52 \\
& $\mathrm{~S}_{2}$ & $157 \pm 1$ & 28 \\
& $\mathrm{~S}_{3}$ & $137 \pm 3$ & 20 \\
\hline
\end{tabular}

Solubilized extracts from tissue homogenates and serum (non-fasted) were used for all the assays. Molecular weight was determined as described in "Materials and Methods." The activity ratios of isoenzymes were calculated from data recorded by the Image Analyzer V1. The same tissue and sera were electrophoresed ten times. The means and SEMs were listed. Isoenzymes were numbered from the cathode side. Abbreviations: L: liver, K: kidney, B: bone, D: duodenum, J: jejunum, I: ileum, S: serum.

fasting. The results are shown in Fig. 6. Total sALP activity decreased after 16 $\mathrm{h}$ of fasting. Supernatant SALP activities decreased after $16 \mathrm{~h}$ of fasting also. The differences between total SALP activity and supernatant sALP activities were not changed; that is, bone-type SALP activities were not changed.

Electrophoretograms of rat $S A L P$ after WGA treatment. The results are shown in Fig. 7. The three bands (S1, S2, S3) of total sALP activity were observed when the serum obtained before fasting was used. The activity of the supernatant prepared by WGA treatment was observed at the positions of S1 and S2. Since the precipitate is a WGA-ALP complex and seems to form a high molecular complex, it was thought that this complex could not enter the gels. Next, we separated the ALP protein from the precipitate (WGA-ALP complex) by GlcNAc epoxyactivated Sepharose 6B column, and performed electrophoresis. The activity band of the ALP protein appeared at the position corresponding to that of S3 by PAGE.

\section{DISCUSSION}

Rat SALP is reportedly constituted by two isoenzymes in normal rats (14), whereas there is another report contradicting this finding (15). Therefore, we first 
Without L-Phe With L-Phe $(20 \mathrm{mM})$

\section{Fasting period (hr)}

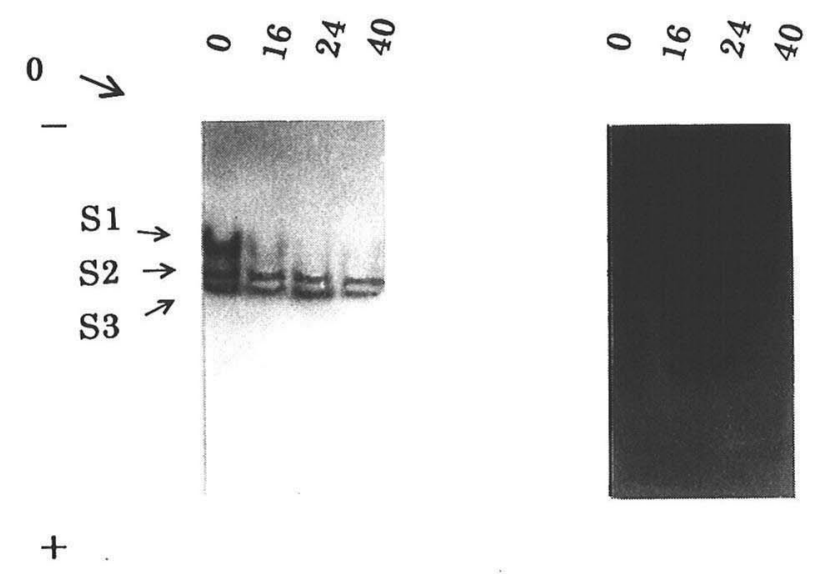

Fig. 5(A). Electrophoretograms of rat SALP during fasting with or without L-Phe.

0 shows the sample application points. The gel was stained for ALP activity.

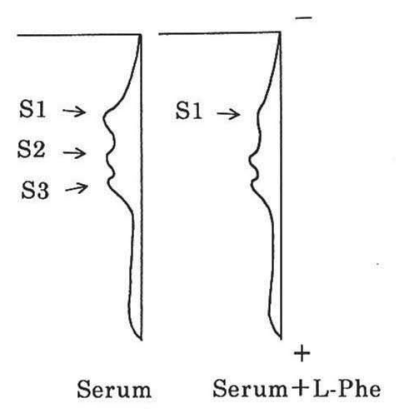

Fig. 5(B). Densitometric scans of electrophoretic patterns of an untreated serum before fasting (left), and of the same serum treated with $20 \mathrm{mM} \mathrm{L-Phe} \mathrm{(right).}$ Densitometric scan was conducted after staining the gel for ALP activity.

examined the isoenzymes of rat sALP by PAGE and obtained three clearly separated bands (S1, S2, S3).

Since S1 was inhibited by L-Phe and its M.W. corresponded to that of an isoenzyme found in the ileum, $\mathrm{S} 1$ was clarified to be derived from the intestine. It is reported that the sugar chains of SALP are not always the same as those of tALP $(26,27)$. This may explain the difference in M.W. between S2 and S3 and the tissue isoenzymes. Therefore, it seemed necessary to identify the derived tissues or organs after cleaving the sugar chains of the ALP isoenzymes for clarification. However, 


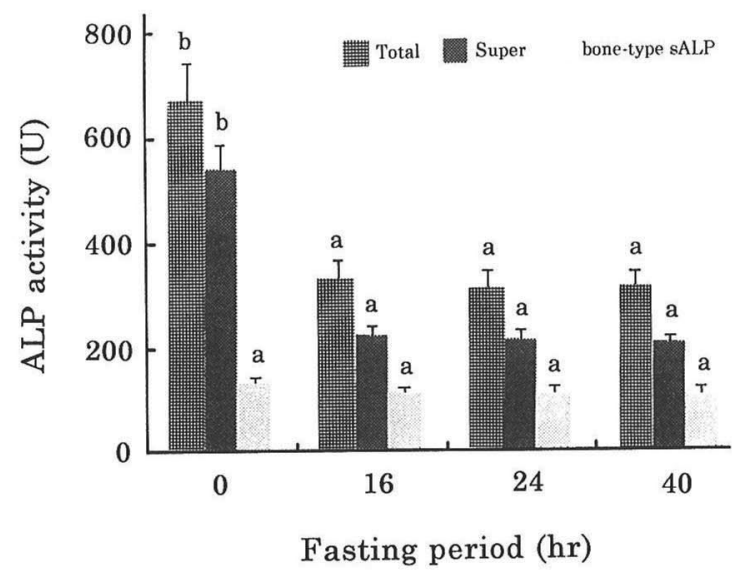

Fig. 6. Changes in total SALP activity and SALP activities precipitated by WGA treatment after fasting. Total: total sALP activity, Super: supernatant sALP activity after WGA treatment, bone-type SALP: ALP activity of the precipitate after WGA treatment. Each value indicates the M \pm SEM of 5-6 rats. Bars without a common superscript letter in the same type of graph are significantly different $(p<0.05)$.

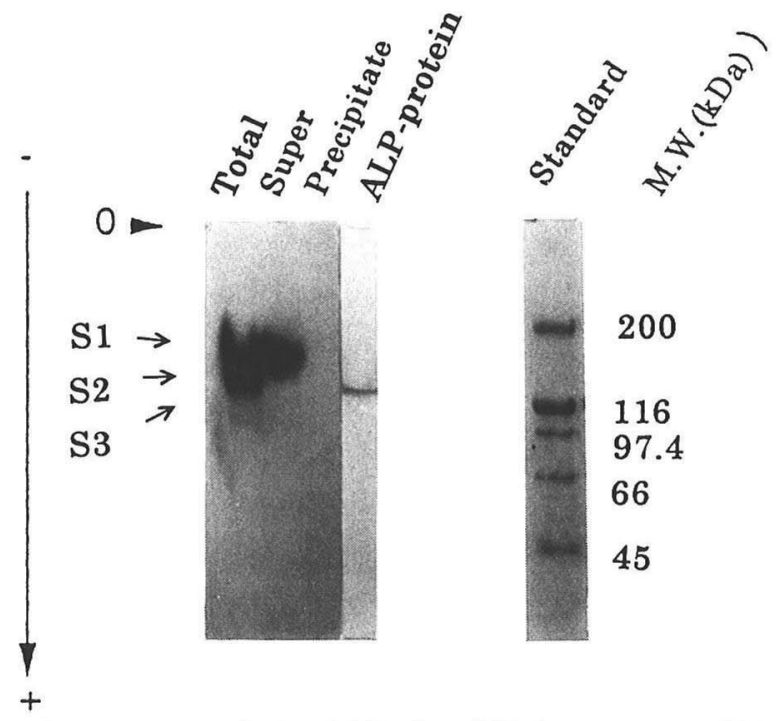

Fig. 7. Electrophoretograms of rat sALP after WGA treatment. Total: total sALP activity band before WGA treatment, Super: supernatant after WGA treatment, Precipitate: precipitate after WGA treatment (before applying column chromatography), ALP-protein: Samples, prepared by WGA treatment and isolated from the WGA-ALP complex by a column of GlcNAc epoxy-activated Sepharose 6B. 
since the activity of the $\mathrm{S} 3$ band obtained by PAGE corresponded to that of the ALP protein precipitated by WGA, and WGA is a selective precipitator of bone-type sALP (8), it seemed possible to postulate that $\mathrm{S} 3$ is derived from bone.

In the study of the tissue identification of three isoenzymes, we used L-Phe, WGA. Fishman et al. reported that L-Phe was a specific inhibitor for human intestinal-type SALP, and that L-HArg was a specific inhibitor for human bone and liver-type $\operatorname{sALP}(6,11,25)$. Amino acids have been used as inhibitors of human sALP and rat sALP isoenzymes $(11,12,28)$. Therefore, this method was also adopted in this study as one of the methods to identify the tissues from which the isoenzymes were derived. Concerning WGA treatment, WGA specifically precipitates bone-type sALP (8), therefore, the WGA-ALP complex is assumed to be bone-type sALP. The precipitate was fractionated by GlcNAc epoxy-activated 6B Sepharose column chromatography, and the fraction obtained, which showed ALP activity, was analyzed by PAGE. The band showing enzymatic activity of the obtained fraction corresponded to S3, therefore, S3 was judged to be bone-type SALP.

Secondly, we examined the changes in enzymatic activity under fasting conditions. The results showed that the activity of $\mathrm{S} 1$ decreased, whereas the activities of S2 and S3 were not affected by fasting.

Righetti and Kaplan (13), and Saini and Posen (16) reported that bone-type SALP activity dominated in rats after fasting. Unakami et al. (3) reported that intestinal-type SALP increased after food intake. However, the time-course changes of SALP isoenzymes under fasting conditions have not been clarified. From our data, it was clarified that the increased ratio of S3 activity (bone-type SALP) under fasting conditions was relative, because there was an absolute decrease of S1 activity. This is the first study on the changes in three ALP isoenzymes in rat serum under fasting conditions.

tALP from intestine is known to have much of its activity released into plasma after feeding (17). The decrease in SALP activity under fasting conditions reflects the decrease in ALP activity in the intestine. Seetharam et al. (29) reported that fat feeding stimulated an increase in the production of intestinal ALP by increasing the mRNA concentration. In the research of insulin-like growth factor-I (IGF-I), the concentration of IGF-I in plasma decreased when rats were subjected to poor nutritional conditions. The synthetic amount of IGF-I mRNA was not changed under these conditions, therefore, the stability of this mRNA is believed to be lower under such conditions (30). In the case of ALP in the intestine, the stability of intestinal ALP mRNA may also be lowered under fasting conditions. In addition, as Warnes et al. (31) postulated that the rise in intestinal ALP after the ingestion of fat was stimulated by the secretion of secretin and cholecystokinin, the changes in secretin and cholecystokinin under fasting conditions may relate to the decrease in intestinal ALP.

In conclusion, under fasting conditions, the activity of S1 decreased while the activities of S2 and S3 remained unchanged. In this study, rat SALP isoenzymes 
were identified by PAGE and the effects of fasting on their activities were clarified.

\section{REFERENCES}

1) Hashimoto, T., Sakurai, T., and Nomoto, S. (1976): Alkaline phosphatase; Reaction mechanism and its physiological significance. Metabolism Disease, 13, 279-283 (in Japanese).

2) Epstein, E., Kiechle, F. L., Artiss, J. D., and Zak, B. (1986): The clinical use of alkaline phosphatase enzymes. Clinics Laboratory Medicine, 6, 491-503.

3) Unakami, S., Hirata, M., Ichinohe, K., Tanimoto, Y., and Iizuka, H. (1989): Separation and quantification of serum alkaline phosphatase isozymes in the rat by affinity electrophoresis. Exp. Anim., 38, 85-88.

4) Moss, D. W., and Whitby, L. G. (1975): A simplified heat-inactivation method for investigating alkaline phosphatase isoenzymes in serum. Clin. Chim. Acta, 61, 63-71.

5) Fishman, W. H., Green, S., and Inglis, N. I. (1962): Organ specific behaviour exhibited by rat intestine and liver alkaline phosphatase. Biochim. Biophys. Acta, 62, 363-375.

6) Fishman, W. H., and Sie, H. G. (1970): L-homoarginine an inhibitor of serum, bone and liver alkaline phosphatase. Clin. Chim. Acta, 29, 339-341.

7) Moss, D. W., and Edward, R. K. (1984): Improved electrophoretic resolution of bone and liver alkaline phosphatases resulting from partial digestion with neuraminidase. Clin. Chim. Acta, 143, 177-182.

8) Rosalki, S. B., and Foo, A. Y. (1984): Two new methods for separating and quantifying bone and liver alkaline phosphatase isoenzymes in plasma. Clin. Chem., 30, 1182-1186.

9) Miura, M., Matsuzaki, H., Baileyes, E. M., Koyama, I., Sakagishi, Y., and Komoda, T. (1989): Difference between human liver and bone type alkaline phosphatase. Clin. Chim. Acta, 180, 177-188.

10) Karmen, C. K., Mayl, P. D., Foo, A. Y., Parhoo, S., and Rosalki, S. B. (1984): Difference between liver and bone type alkaline phosphatase. J. Clin. Pathol., 37, 212217.

11) Pickering, C. E., and Pickering, R. G. (1978): Studies of rat alkaline phosphatase, I. Development of methods for detecting isoenzymes. Arch. Toxicol., 39, 249-266.

12) Pickering, C. E., and Pickering, R. G. (1978): Studies of rat alkaline phosphatase, II. Some application of the methods for detecting the isoenzymes of plasma alkaline phosphatase in rats. Arch. Toxicol., 39, 267-287.

13) Righetti, A. B., and Kaplan, M. M. (1971): The origin of serum alkaline phosphatase in normal rats. Biochim. Biophys. Acta, 230, 504-509.

14) Kaschnitz, R., Patsch, J., and Peterlic, M. (1968): Isoenzyme der Alkalischen Phosphatase in der loslichen Phase von Rattenleberzellen. Eur. J. Biochem., 5, 51-54.

15) Horiguchi, M., Imaizumi, T., and Hagiwara, M. (1977): Serum alkaline phosphatase of patient with tumor and tumor bearing rat. Physico-Chemical Biology, 20, 335 (in Japanese).

16) Saini, P. K., and Posen, S. (1969): The origin of serum alkaline phosphatase in the rat. Biochim. Biophys. Acta, 177, 42-49.

17) Young, G. P., Friedman, S., Yedlin, S. T., and Alpers, D. H. (1981): Effect of fat 
feeding on intestinal alkaline phosphatase activity in tissue and serum. Am. J. Physiol., 241, 461-468.

18) Saini, P. K., and Done, J. (1972): The diversity of alkaline phosphatase from rat intestine isolation and purification of the enzyme(s). Biochim. Biophy. Acta, 258, 147153.

19) Bessey, O. A., Lowry, O. H., and Brock, M. J. (1946): A method for rapid determination of alkaline phosphatase with five cubic millimeters of serum. J. Biol. Chem., 164, 321-329.

20) Fishman, W. H., Green, S., and Inglis, N. I. (1963): L-phenylalanine: an organ specific inhibitor of human intestinal alkaline phosphatase. Nature, 198, 685-686.

21) Vretblad, P. (1976): Purification of lectins by biospecific affinity chromatography. Biochim. Biophys. Acta, 434, 169-171.

22) Lowry, O. H., Rosebrough, N. J., Farr, A. L., and Randall, R. J. (1951): Protein measurement with the Folin phenol reagent. J. Biol. Chem., 193, 265-275.

23) Komoda, T., Kumegawa, M., Yajima, T., Tamura, G., and Alpers, D. H. (1984): Induction of rat hepatic and intestinal alkaline phosphatase activity produced by bile from bile duct-ligated animals. Am. J. Physiol., 246, 393-400.

24) Fishman, W. H., Inglis, N. I., and Gosh, N. K. (1968): Distinctions between intestinal and placental isoenzyme of alkaline phosphatase. Clin. Chim. Acta, 19, 71-79.

25) Komoda, T., Hokari, M., Sonoda, M., Sakagishi, Y., and Tamura, T. (1982): LPhenylalanine inhibition of human alkaline phosphatases with $p$-nitrophenyl phosphate as substrate. Clin. Chem., 28, 2426-2428.

26) Komoda, T., Koyama, I., Nagata, A., Sakagishi, Y., Kurata, M., and Kumegawa, M. (1986): A possible mechanism of induction and translocation into blood stream of rat alkaline phosphatase activity by bile duct ligation. Arch. Biochem. Biophys., 251, 323335.

27) Unakami, S., Komoda, T., and Sakagishi, Y. (1990): Translocation of intestinal alkaline phosphatase in streptozotocin-induced diabetic rats. Int. J. Biochem., 22, $1325-1331$.

28) Nomoto, S. (1985): Alkaline or acid phosphatase and alkaline phosphatase isoenzyme, in Rinsyo Kensa Teiyo (Kanai's Manual of Clinical Laboratory Method), ed. by Kanai, I. and Kanai, M., Kanahara \& Co., Tokyo, pp. 495-505 (in Japanese).

29) Seetharam, S., Ovitt, C., Strauss, A. W., Rubin, D., and Alpers, D. H. (1987): Fat feeding stimulates only one of the two rat intestinal membranous and secreted alkaline phosphatase. Biochem. Biophys. Res. Commun., 145, 363-368.

30) Noguchi, T. (1996): Protein metabolism and IGF-I, in Molecular Nutrition, ed. by Tanaka, T., Noguchi, T., and Mutou, Y., Kenpakusha Co., Ltd., Tokyo, pp. 101-122 (in Japanese).

31) Warnes, T. W., Hine, P., and Kay, G. (1974): The action of secretin and pancreozymin on small intestine alkaline phosphatase. Gut, 15, 39-47. 